Physica A 168 (1990) 919-941

North-Holland

\title{
RESCALED MEAN SPHERICAL APPROXIMATION FOR COLLOIDAL MIXTURES
}

\author{
H. RUIZ-ESTRADA
}

Departamento de Física, Centro de Investigación y de Estudios Avanzados del IPN, Apartado Postal 14-740, 07000 México, D.F., Mexico

\section{MEDINA-NOYOLA}

Instituto de Física "Manuel Sandoval Vallarta", Universidad Autónoma de San Luis Potosí, Apartado Postal 629, 78000 San Luis Potosi, S.L.P., Mexico

\section{G. NÄGELE}

Department of Nuclear Engineering, University of Michigan, Ann Arbor, MI 48109, USA

Received 10 May 1990

In this work, the rescaled mean spherical approximation (RMSA) for colloidal mixtures interacting via a DLVO-type potential is developed, and its application to suspensions of highly charged macroions is illustrated. For this purpose we introduce a simple scheme to solve the mean spherical approximation (MSA) for Yukawa mixtures with factorized coupling parameters. This scheme consists of the mapping of the Yukawa system onto a corresponding primitive model system. Such a correspondence is used as a device for the calculation of the static structure functions of the original Yukawa mixture. Within this scheme, a straightforward implementation of the rescaling procedure is performed, which allows for the calculation of partial structure factors in strongly intcracting mixtures. The rescaling procedure we use is an extension of that introduced by Hansen and Hayter for monodisperse suspensions. The structure factors obtained with the rescaled mean spherical approximation compare well with computer simulation results. The advantages and limitations of the RMSA are also discussed in some detail.

\section{Introduction}

The rescaled mean spherical approximation (RMSA) for the one component macrofluid (OCM) model of suspensions of charged spherical particles, micellar solutions, etc., has found widespread application in the description of the structural properties of these systems. This is due to the practical simplicity resulting from the analytic solution of the mean spherical approximation for Yukawa potentials and from the use of the rescaling idea introduced by Hansen and Hayter [1]. The qualitative and quantitative accuracy of the static structure 
predicted by this approximation has been discussed rather extensively. It is found in particular $[2,3]$ that the RMSA results compare generally well with computer simulation data and with the results of other more elaborate and purely numerical (but reputedly more precise) integral equations. Furthermore, it is now clear that a large amount of experimental data can be represented quite successfully in terms of this approximate scheme. In fact, even near the disorder-order phase transition, where the straight comparison of the RMSA results with the computer-simulated data is not quantitatively good enough, the RMSA has been found to provide a convenient fitting device, from which the static structure factor at large and small wavelengths can be extrapolated [2]. The knowledge of this information is important for the calculation of dynamic properties, especially of transport coefficients.

Unfortunately, a monodisperse suspension is to a large extent an exceptional system from a practical point of view. Therefore, it is desirable to extend the RMSA scheme to multicomponent suspensions of particles interacting by DLVO-type potentials, i.e., a hard-sphere plus a Yukawa pair potential with factorized coupling parameters of the form

$$
\frac{u_{\alpha \beta}(r)}{k_{\mathrm{B}} T}=A_{\alpha r} A_{\beta} \frac{\mathrm{e}^{-z r}}{r} .
$$

Here $A_{\alpha x}$ denotes the coupling amplitude of particles of species $\alpha$ and $z$ is the screening parameter. An important example is the long-ranged part of the DLVO potential for mixtures, in which case

$$
A_{\alpha}^{\mathrm{DLVO}}=L_{\mathrm{B}}^{1 / 2} Z_{\alpha} \frac{\mathrm{e}^{\kappa \sigma_{\alpha / 2} / 2}}{1+\sum_{2}^{1} \kappa \sigma_{\alpha}} .
$$

In this equation $Z_{\alpha}$ and $\sigma_{\alpha}$ are, respectively, the macroion charge number and diameter of species $\alpha, L_{\mathrm{B}}$ is the Bjerrum length, i.e., $L_{\mathrm{B}} \equiv \mathrm{e}^{2} / \epsilon k_{\mathrm{B}} T$ and $\kappa$ is the Debye screening parameter due to the ionic strength of the counterions and salt ions. This particular form of the potential of mean force can be obtained from a linearized Poisson-Boltzmann equation for an infinitely dilute suspension at large particle distance $r$.

The solution of the mean spherical approximation (MSA) for multicomponent Yukawa mixtures has been reported by Blum and Hoye [4]. For its practical use, however, a coupled set of algebraic equations, which possesses in general several solutions, has to be solved [5]. As it has been shown by Pastore [6], only one of such solutions is physical. Its identification, however, is still rather cumbersome [5,6]. One could also mention Ginoza's [7] demonstration that Blum's solution can be largely simplified for Yukawa mixtures with factorized coupling constants, such as in eq. (1.1). In his solution only a single non-linear equation in one parameter has to be solved. Still, the form of this 
non-linear equation is rather complicated, and it is not clear if it has in general only one solution. Thus, one is still left with the task of determining the physical root.

In contrast to this, the MSA solution (also found by Blum) for the primitive model (PM), i.e., charged hard spheres, is extraordinarily simple [8-11]. Here, too, a non-linear equation in a single parameter, $\Gamma$, has to be solved. However, one can show that this equation has only one real positive solution, which is the physical onc [10]. As we demonstrate in this paper, this fact can be used to simplify the task of determining the physical MSA structure functions of a Yukawa mixture. Thus, the first purpose of this work is to establish a method of solution of the MSA for Yukawa mixtures based on the transformation of the original problem into the mathematically equivalent (but much easier) problem of determining the MSA structure functions of a corresponding primitive model system.

The second task of the present work consists in the application of this method of solution of the MSA for Yukawa mixtures, to the implementation of the rescaling procedure, originally suggested by Hansen and Hayter [1] for monodisperse suspensions.

This paper is organized as follows. In section 2, we extend to mixtures an important observation made by Belloni [12] for monodisperse systems. Thus, we show that the effective pair potential between macroions in a primitivemodel representation of a polydisperse suspension with point-like small ions (counterions and added salt ions) is of the Yukawa type as shown in eq. (1.1). In section 3 we use this observation to establish the equivalence between the MSA solution for an $m$-component Yukawa mixture and The MSA solution of a corresponding $(m+1)$-component primitive model. There we also illustrate the use of the resulting algorithm by calculating the MSA structure functions of a binary Yukawa mixture. In section 4 we explain how this algorithm can be combined with the rescaling procedure for the mean spherical approximation, thus defining the "rescaled mean spherical approximation" (RMSA) for Yukawa mixtures. Here we also illustrate the resulting approximation by calculating the structural properties of a bidisperse suspension of highly charged macroions, comparing our results to computer simulation data. Furthermore, in that section we comment on the range of applicability of the RMSA for mixtures. The main conclusions of the present work are summarized in section 5 .

\section{Effective pair potentials in a macroion mixture}

A long-standing problem in colloid science has been the determination of the 
effective pair potentials between charged spherical particles in suspension. The basic model for the description of this effective interaction is the primitive model, where the macroparticles, the counterions, and salt ions are all represented as charged hard spheres in a dielectric continuum (the solvent). The classical work of Derjaguin, Landau, Verwey, and Overbeek led to the first description of such an effective pair potential in the limit of infinite dilution of macroparticles and zero size of the small ions. After several approximations, their work leads to the particular case of eq. (1.2) corresponding to equal-sized spheres. More recently, such a particular expression for the effective pair potential has been taken as the starting point in the description of the structural properties of interacting suspensions at finite concentrations, in the spirit of the so-called "one component macrofluid" (OCM) model. Thus, the structural propertics of a monodisperse suspension at finite concentrations can be calculated in two different manners. The first is the application of approximations developed in the theory of simple liquids to the calculation of the structural properties of this effective OCM system [1-3]. The second, conceptually more fundamental, but generally more involved in practice. is the usc of similar approximations directly at the primitive-model level [12 18]. The relationship between these two approaches has been clarified, at least within the framework of the mean spherical approximation, starting with the work of Medina-Noyola and McQuarrie [15]. In the most recent discussion of this subject, Belloni [12] has made the observation that there is a close relationship between the MS $\Lambda$ solution for the two-component primitive model (macroions and point-like counterions) and the MSA solution for a corresponding OCM model. In what follows we extend this observation to multicomponent mixtures of macroions. Thus, we demonstrate that, within the MSA, the effective pair potential between two macroions in an $(m+l)$-component primitive model system with $m$ macroion species plus $l$ species of point-like small ions (counterions and added salt ions), is of the Yukawa type in eq. (1.1), with the screening parameter determined by the ionic strength of the small ions. In the following section we shall profit from these results by demonstrating that the MSA solution for an $m$-component Yukawa system with factorized coupling parameters is most easily determined by solving the MSA for a corresponding $(m+1)$-component primitive model system.

Let us first consider for simplicity a $(m+1)$-component primitive model consisting of $m$ species of macroions plus their point-like counterions (species $(m+1))$. In the MSA, the radial distribution functions $g_{i j}(r)$ for this system are obtained by solving the Ornstein-Zernike equation

$$
h_{i j}(r)=c_{i j}(r)+\sum_{k=1}^{m+1} n_{k} \int h_{i k}\left(\left|r-r^{\prime}\right|\right) c_{k j}\left(r^{\prime}\right) \mathrm{d}^{3} r^{\prime}
$$


with the following closure relations:

$$
\begin{aligned}
& c_{i j}(r)=-L_{\mathrm{B}} \frac{Z_{i} Z_{j}}{r}, \quad r>\sigma_{i j} \equiv \frac{1}{2}\left(\sigma_{i}+\sigma_{j}\right), \\
& h_{i j}(r)=-1, \quad r<\sigma_{i j} .
\end{aligned}
$$

Herc, $c_{i j}(r)$ are the direct corrclation functions and $h_{i j}(r)=g_{i j}(r)-1$ are the total correlation functions. Also, $n_{i}, \sigma_{i}$, and $Z_{i}$ are, respectively, the number density, diameter, and charge (in units of the elementary charge $e$ ) of species $i$, and $L_{\mathrm{B}}$ is the Bjerrum length. Eqs. (2.1)-(2.3) constitute the MSA closure relation. Other closure relations have been discussed in the literature, but only the MSA allows for a simple analytic calculation of the correlation functions of the primitive model. As has been demonstrated by Blum and Hoye [8-11], all correlation functions can be expressed in terms of only a single parameter, $\Gamma$, whose value is obtained by solving the algebraic equation (see ref. [10])

$$
\Gamma^{2}=\pi L_{\mathrm{B}} D(\Gamma)
$$

with

$$
D(\Gamma)=\frac{\kappa^{2}}{4 \pi L_{\mathrm{B}}}+\sum_{\alpha=1}^{m} n_{\alpha}\left(Y_{\alpha}\right)^{2},
$$

where

$$
Y_{\alpha}=\frac{1}{1+\Gamma \sigma_{\alpha}}\left(Z_{\alpha}-\frac{\pi}{2 \Delta} \sigma_{\alpha}^{2} \frac{\sum_{\gamma=1}^{m} n_{\gamma} \sigma_{\gamma} Z_{\gamma} /\left(1+\Gamma \sigma_{\gamma}\right)}{1+(\pi / 2 \Delta) \sum_{\gamma=1}^{m} n_{\gamma} \sigma_{\gamma}^{3} /\left(1+\Gamma \sigma_{\gamma}\right)}\right)
$$

In these equations

$$
\kappa^{2}=4 \pi L_{\mathrm{B}} n_{\mathrm{a}} Z_{\mathrm{s}}^{2},
$$

where $\kappa$ is the Debye screening constant, due to the point-like counterions, and $\Delta=1-\frac{1}{6} \pi \Sigma_{\gamma=1}^{m} n_{\gamma} \sigma_{\gamma}^{3}$. From here on we will label the macroion species by Greek subindices, whereas the counterions, species $(m+1)$, will be labelled by s. Eq. (2.4) allows for only one, positive valued, real solution since $D(\Gamma)$ is a monotonously decreasing function of its argument. This solution is easily obtained by using, e.g., the Newton-Raphson algorithm. Once this parameter $I$ is determined, the static structure factors are easily calculated using the 
analytic expressions reported by Blum and Hoye [8-11]. The OrnsteinZernike equation for the $(m+1)$ species in eq. (2.1) can be contracted to eliminate the explicit reference to the correlation functions involving the counterions. This contraction procedure leads to the following "effective" Ornstein-Zernike equation:

$$
h_{\alpha \beta}(r)=c_{\alpha \beta}^{\mathrm{cllf}}(r)+\sum_{\gamma}^{m} n_{\gamma} \int h_{\alpha \gamma \gamma}\left(\left|\boldsymbol{r}-\boldsymbol{r}^{\prime}\right|\right) c_{\gamma \beta}^{\mathrm{cll}}\left(r^{\prime}\right) \mathrm{d}^{3} r^{\prime}
$$

which contains only the total correlation functions between the macroions. This contraction procedure was first discussed by Adelman [20], and employed by Belloni [12] in the present context, in reference to a monodisperse macroion suspension. The form of the direct correlation functions $c_{\alpha \beta \beta}^{\text {eff }}(r)$ of the effective $m$-component system is determined from the condition that $h_{\alpha \beta}(r)$ in eq. (2.8) are identical with the macroion-macroion total correlation functions in eq. (2.1). From this it follows that

$$
C_{\alpha \beta}^{\mathrm{tH}}(q)=C_{\alpha \beta}(q)+\frac{C_{\alpha s}(q) C_{\beta,}(q)}{1-C_{s}(q)}
$$

where $C_{\alpha \beta}(q), C_{\alpha s}(q)$, and $C_{\alpha \beta}^{\mathrm{ctI}}(q)$ are, respectively, the Fourier transforms of the direct correlation functions $\left(n_{\alpha r} n_{\beta}\right)^{1 / 2} c_{\alpha \beta \beta}(r), \quad\left(n_{\alpha r} n_{4}\right)^{1 / 2} c_{\alpha,}(r)$, and $\left(n_{\alpha} n_{\beta}\right)^{1 / 2} c_{\alpha \beta}^{\mathrm{effi}}(r)$.

In a next step we approximate the direct correlation functions between the point-like counterions by their Debye-Hückel approximation (i.e., MSA with $\sigma_{\mathrm{s}}=0$ ),

$$
c_{\mathrm{ss}}(r)=-L_{\mathrm{B}} \frac{Z_{s}^{2}}{r}, \quad r>0 .
$$

Then, from eq. (2.9), it follows that

$$
\begin{aligned}
C_{\alpha \beta}^{\mathrm{cff}}(q)= & C_{\alpha \beta}^{\prime}(q)+C_{\alpha \varsigma}^{\prime}(q) C_{\beta \mathrm{s}}^{\prime}(q) \\
& -\frac{\left(4 \pi L_{\mathrm{B}}\right)^{1 / 2}}{q^{2}+\kappa^{2}}\left[n_{\alpha}^{1 / 2} Z_{\alpha}+n_{\mathrm{s}}^{1 / 2} Z_{\mathrm{s}} C_{\alpha s}^{\prime}(q)\right]\left[n_{\beta}^{1 / 2} Z_{\beta}+n_{s}^{1 / 2} Z_{\mathrm{s}} C_{\beta \mathrm{s}}^{\prime}(q)\right],
\end{aligned}
$$

where

$$
C_{\alpha \beta}^{\prime}(q)=C_{\alpha \beta}(q)+4 \pi L_{\mathrm{B}}\left(n_{i x} n_{\beta}\right)^{1 / 2} Z_{\alpha} Z_{\beta} / q^{2}
$$


is the short-ranged part of $C_{\alpha \beta}(q)$. A similar definition holds for the shortranged part, $C_{\alpha \mathrm{s}}^{\prime}(q)$, of the weighted macroion-counterion direct correlation functions. From eq. (2.11) it follows in the limit of large particle distance $r$ that

$$
c_{\alpha \beta}^{\mathrm{eff}}(r) \sim-K_{\alpha \beta} \frac{\mathrm{e}^{-\kappa r}}{r} \equiv-\frac{1}{k_{\mathrm{B}} T} u_{\alpha \beta}^{\mathrm{eff}}(r)
$$

with coupling parameters $K_{\alpha \beta}$, whose value depends on the specific form used for the macroion-counterion direct correlation functions. Eq. (2.13) defines the effective pair potentials $u_{\alpha \beta}^{\text {eff }}(r)$ between the macroions. These effective potentials are found to be of the Yukawa type, with the screening constant $\kappa$ given by eq. (2.7).

The next step is to choose an approximation for the macrion-counterion direct correlations functions. In the framework of monodisperse suspensions, several different closure relations for these correlation functions have been discussed. Beresford-Smith et al. [16] used the HNCA closure relations in addition to the so-called jellium approximation for the macroion-macroion correlations. This approximation treats all the macroions, except one, as being smeared out. It preserves overall charge neutrality, but it neglects contributions of the other macroions to the direct correlations between a given macroion and its counterion double layer. The coupling parameter of the effective pair potential is obtained in this approximation from the numerical solution of a Poisson-Boltzmann type equation. Belloni [12], on the other hand, used the MSA closure for both the macroion-macroion and macroioncounterion direct correlation functions. This is a better approximation regarding the influence of the macroion distribution, on the macroion-counterion correlations. However, the accumulation of the counterions close to the surface of a highly charged macroion is better described by the non-linear HNCA closure.

In extending Belloni's approach to $m$-components of macroions, we treat both $c_{\alpha \beta}(r)$ and $c_{\alpha s}(r)$ within the MSA. Then, as Hiroike has shown, the short-ranged part of $c_{\alpha s}(r)$ is given by the following simple expression (see eq. (15) of ref. [10] and eq. (3.12) of ref. [11]):

$$
c_{\alpha \mathrm{s}}^{\prime}(r)=\left\{-\frac{1}{\Delta}+L_{\mathrm{B}} Z_{\alpha} Z_{\beta}\left[\frac{2}{\sigma_{\alpha}}\left(\frac{Y_{\alpha}}{Z_{\alpha}}-1\right)+\frac{1}{r}\right]\right\} \theta\left(\frac{1}{2} \sigma_{\alpha}-r\right),
$$

with $Y_{\alpha}$ defined in eq. (2.6). By observing that the short-ranged part of the direct correlation functions in MSA is identically zero outside the hard-core distance, the following important result is obtained:

$$
-c_{\alpha \beta}^{\text {eff }}(r) \equiv \frac{u_{\alpha \beta}^{\text {eff }}(r)}{k_{\mathrm{B}} T}=K_{\alpha} K_{\beta} \frac{\mathrm{e}^{-\kappa r}}{r}, \quad r>\sigma_{\alpha \beta},
$$


with the coupling amplitudes $K_{r x}$ given by

$$
K_{\alpha}=L_{\mathrm{B}}^{1 / 2} Z_{\alpha}\left\{\cosh \left(\frac{1}{2} \kappa \sigma_{\alpha}\right)+U_{\alpha}\left[\frac{1}{2} \kappa \sigma_{\alpha} \cosh \left(\frac{1}{2} \kappa \sigma_{\alpha}\right)-\sinh \left(\frac{1}{2} \kappa \sigma_{\alpha}\right)\right]\right\},
$$

where

$$
U_{c t}=\frac{3}{\Delta\left(\frac{1}{2} \kappa \sigma_{c r}\right)^{3}} \sum_{\gamma=1}^{m} \phi_{\gamma}\left(\frac{\sigma_{\alpha}}{\sigma_{\gamma}}\right)^{3} \frac{Z_{\gamma}}{Z_{\alpha r}}+\frac{1}{\left(\frac{1}{2} \kappa \sigma_{c r}\right)}\left(\frac{Y_{\alpha v}}{Z_{c r}}-1\right) .
$$

In the above expressions, $\phi_{c r}=\frac{1}{6} \pi n_{c r} \sigma_{c \alpha}^{3}$ is the volume fraction of species $\alpha$. Notice that $K_{\alpha}$ depends on the number densities, charges, and diameters of all species, including the counterions. Its value is obtained once the parameter $I$ is determined from eq. (2.4). Notice also that here the effective pair potential is of the DLVO type, i.e., a Yukawa potential with factorized coupling parameters $K_{\alpha \beta}=K_{\alpha} K_{\beta}$. In the limit of vanishing macroion concentration, eq. (2.15) reduces to the DLVO potential given in eqs. (1.1), (1.2), thus extending the result of Medina-Noyola and McQuarrie [19]. In the special case of only one species of macroions, eq. (2.15) reduces to the expression given by Belloni [12]. Let us mention, however, that Senatore and Blum [15] had previously considered the contraction of a two-component primitive model into a onecomponent macroion system at finite concentration, within the MSA. From their numerical calculations of the effective macroion-macroion direct correlation function, they conjectured a Yukawa form for this effective pair potential. Our results extend Belloni's verification of this conjecture.

As a final remark, let us emphasize once again that our present derivation involved for simplicity only one species of small ions, namely, the counterions. This derivation, however, can be extended to the case in which added salt is considered at the primitive-model level. In this manner, one finds that the contraction of a $(m+l)$-component primitive model with $m$ macroion species and $l$ species of point-like small ions, into an effective $m$-component macrofluid system, yields the same results as above, except that the Debye screening parameter $\kappa$ is determined not by eq. (2.7), but by the ionic strength of all the species of small ions, i.e., $\kappa^{2}=4 \pi L_{\mathrm{B}} \Sigma_{j-m+1}^{m+1} n_{j} Z_{j}^{2}$.

\section{MSA for the primitive model, a device to solve the MSA for Yukawa mixtures}

The results of the previous section allow for the following interpretation (sec ref. [15] for the particular case when $m=1$ ). The MSA macroion-macroion 
radial distribution functions $g_{\alpha \beta}(r)$ of a $(m+1)$-component PM with point-like counterions may be obtained most directly from the analytic solution of eqs. (2.1)-(2.3) (which, in addition, would also provide the macroion-counterion correlations). However, these correlation functions also satisfy eq. (2.8) with $c_{\alpha \beta}^{\text {eff }}(r)$ given by eq. (2.15). Thus, they are the solution of the Ornstein-Zernike equation

$$
h_{\alpha \beta}(r)=c_{\alpha \beta}(r)+\sum_{\gamma=1}^{m} n_{\gamma} \int h_{\alpha \gamma}\left(\left|r-r^{\prime}\right|\right) c_{\gamma \beta}\left(r^{\prime}\right) \mathrm{d}^{3} r^{\prime}
$$

of a corresponding $m$-component Yukawa system with closure relations

$$
c_{\alpha \beta}(r)=-A_{\alpha} A_{\beta} \frac{\mathrm{e}^{-z r}}{r}, \quad r>\sigma_{\alpha \beta},
$$

and

$$
h_{\alpha \beta}(r)=-1, \quad r<\sigma_{\alpha \beta},
$$

provided that the Yukawa coupling parameters $A_{\alpha}$, and screening constant $z$, are given by

$$
A_{\alpha}=K_{\alpha}\left(Z_{1}, \ldots, Z_{m}, Z_{\mathrm{s}}\right)
$$

and

$$
z=\kappa\left(Z_{1}, \ldots, Z_{m}, Z_{\mathrm{s}}\right),
$$

where the functions $K_{\alpha}$ and $\kappa$ were defined in eqs. (2.16) and (2.7), respectively. Notice, however, that the Debye screening constant $\kappa$ depends indirectly also on the macroion charges via the overall charge-neutrality condition. Solving eqs. (3.1)-(3.3) to determine the macroion-macroion correlations is, of course, an unnecessary complication of a relatively simple problem, since the radial distribution functions of the primitive model are much easier to calculate using directly the analytic solution [8-11] of eqs. (2.1)-(2.3), than the solution of eqs. (3.1)-(3.3) discussed in refs. [4, 5].

The inverse interpretation of this observation is, of course, a more profitable way of using these results, provided that we are interested in obtaining the MSA structure functions of an arbitrary $m$-component Yukawa system of the type in eq. (1.1) with diameters $\sigma_{\alpha}$ and number densities $n_{\alpha}$, for given parameters $A_{\alpha}(\alpha=1,2, \ldots, m)$ and $z$. In this case, the $(m+1)$ equations (3.4), (3.5) could be numerically inverted to determine the parameters $Z_{\alpha}$ and 
$Z_{\mathrm{s}}$ of a corresponding primitive model with the same macroion diameters and number densities as for the Yukawa system, and with the number density of the point-like counterions determined by the electroneutrality condition. (Notice then that the counterion charge number $Z_{\mathrm{s}}$ and number density $n_{\mathrm{s}}$ can be trivially expressed in terms of the macroion charge numbers $Z_{1}, \ldots, Z_{m}$ by using eq. (3.5) and the electroneutrality condition. It is, therefore, only necessary to solve the $m$ equations (3.4) numerically for the $m$ unknowns $Z_{1}, \ldots, Z_{m}$ ). The resulting solution defines the primitive model system whose MSA macroion-macroion structure functions are identical to the MSA structure functions of the originally given $m$-component Yukawa mixture. Thus, the latter can be calculated quite conveniently from the analytic solution of the MSA for the corresponding primitive model, since in this manner there is no ambiguity concerning the physical solution. This is to be contrasted with the available methods of solution of the MSA for the actual multicomponent Yukawa system (eqs. (3.1)-(3.3)), which is comparatively more involved because, precisely, of the practical difficulties to single out the physical solution (as discussed by Arrieta et al. [5] and Pastore [6]).

Notice that in general the physical significance of the primitive model thus associated to a given Yukawa mixture is not particularly obvious. Thus, for the moment such a primitive model plays here no other role than that of a convenient mathematical device to simplify an otherwise rather cumbersome numerical problem. Thus, we should not be concerned if, for example, the resulting "charge" numbers $Z_{\alpha}$ are not exact integers.

To illustrate the use of this scheme, we calculate the partial structure functions $g_{\alpha \beta}(r)$ and $S_{\alpha \beta}(q)$ for a binary Yukawa mixture with parameters $n_{1}=4.03 \times 10^{18} \mathrm{~cm}^{-3}, \quad n_{2}=5.35 \times 10^{17} \mathrm{~cm}^{-3}, \quad \sigma_{1}=50 \AA, \quad \sigma_{2}=70 \AA, \quad a_{1}=$ $54.14 \AA^{1 / 2}, A_{2}=166.96 \AA^{1 / 2}$ and $z=6.77 \times 10^{-2} \AA^{1}$. These parameters are typical of a concentrated miccllar solution (whose composition was chosen in such a way that both species contribute equally to the scattered intensity). The three equations (3.4), (3.5) are first numerically solved for the parameters $Z_{1}$, $Z_{2}$ and $Z_{\mathrm{s}}$ of the correspondent three-component primitive model. We find $Z_{1}=8.04, Z_{2}=15.44$ and $Z_{\mathrm{s}}=-1.25$.

Let us notice also that, for given Yukawa parameters $A_{\alpha}$ and $z$, besides the charges $Z_{\alpha}$ of the corresponding primitive model, one could also define "DLVO charge"-numbers, $Z_{\alpha}^{\text {DLVO }}$, as

$$
Z_{\alpha}^{\mathrm{DLVO}} \equiv L_{\mathrm{B}}^{-1 / 2} A_{\alpha} \mathrm{e}^{-z \sigma_{\alpha} / 2}\left(1+\frac{1}{2} z \sigma_{\alpha}\right),
$$

which is eq. (1.2), with $A_{\alpha}^{\text {DLvo }}=A_{\alpha}$ and $\kappa=z$. For the system in our example, we find that $Z_{1}^{\text {DLVO }}=10$ and $Z_{2}^{\text {DLVO }}=19.6$. Thus, in this case we observe that $Z_{\alpha}<Z_{\alpha}^{\text {DLVO }}$. 
Fig. 1a shows the three partial structure factors $S_{\alpha \beta}(q)$ of the Yukawa mixture of our example, obtained in the manner explained above. We have checked these results against the direct calculation of the structurc functions using Blum and Hoye's solution [4] of the two-component Yukawa-system as elaborated by Arrieta et al. [5] and Pastore [6] and found, of course, complete agreement. To calculate the corresponding radial distribution functions $g_{\alpha \beta}(r)$ in fig. 1b, a Fourier sine transform algorithm was used, and an asymptotic correction is included to obtain $g_{\alpha \beta}(r)$ accuratcly everywhere, including in the neighborhood of the contact distance $r=\sigma_{\alpha \beta}$.

There is still the question whether the $m$ equations (3.4) can always be solved for PM parameters $\left\{Z_{1}, \ldots, Z_{m}\right\}$, for arbitrary Yukawa coupling amplitudes $A_{\alpha}$, and if so, whether the set $\left\{Z_{1}, \ldots, Z_{m}\right\}$ is unique. For the Yukawa systems we considered we did always find a set of PM parameters, and hence, we conjecture that corresponding PM parameters can always be found, although we cannot provide a rigorous proof. Our conjecture is supported, however, by the consideration of limiting cases: in the infinite-dilution limit, $n_{\alpha} \rightarrow 0$, where we have that $K_{\alpha} \rightarrow K_{\alpha}^{\text {DLVO }}$, and in the limit $\alpha_{\alpha} \rightarrow 0$, of point-like macroions, a one-to-one correspondence between the two sets $\left\{A_{1}, \ldots, A_{m}\right\}$ and $\left\{Z_{1}, \ldots, Z_{m}\right\}$ is guaranteed.

On the other hand, the question concerning the uniqueness of the set $\left\{Z_{1}, \ldots, Z_{m}\right\}$ is in fact irrelevant for our present purpose, in which the PM is being used as a mere mathematical device. Thus, even if there were more than one set of PM parameters solving eqs. (3.4), all of the corresponding PM systems must be such that their macroion-macroion correlation functions coincide, and are identical to those of the $m$-component Yukawa mixture. However, since we expect that different charges in the PM give rise to different MSA structure functions, it is resasonable to expect that the set $\left\{Z_{1}, \ldots Z_{m}\right\}$ is in fact unique.

The main purpose of this section was to establish a convenient algorithm to solve the MSA for fluid mixtures of particles interacting through hard-sphere plus arbitrary Yukawa tails of the type in eq. (1.1). Here, however, we shall not attempt to determine the general degree of accuracy of the MSA structural propcrties as compared, for example, with computer simulation results. We know, in fact, that for dilute suspensions of highly charged particles, this approximation may lead to the unphysical result that $g_{\alpha \beta}(r)$ becomes negative near contact. To overcome this difficulty, the rescaling procedure will be implemented in the following section. However, in the opposite regime, i.e., for weakly charged suspensions at high volume fractions, such as in our example in fig. 1, the MSA is expected to be a reasonably accurate approximation, since in this regime the electrostatic effects can be considered as a perturbation with respect to the uncharged hard-sphere reference mixture. Let 

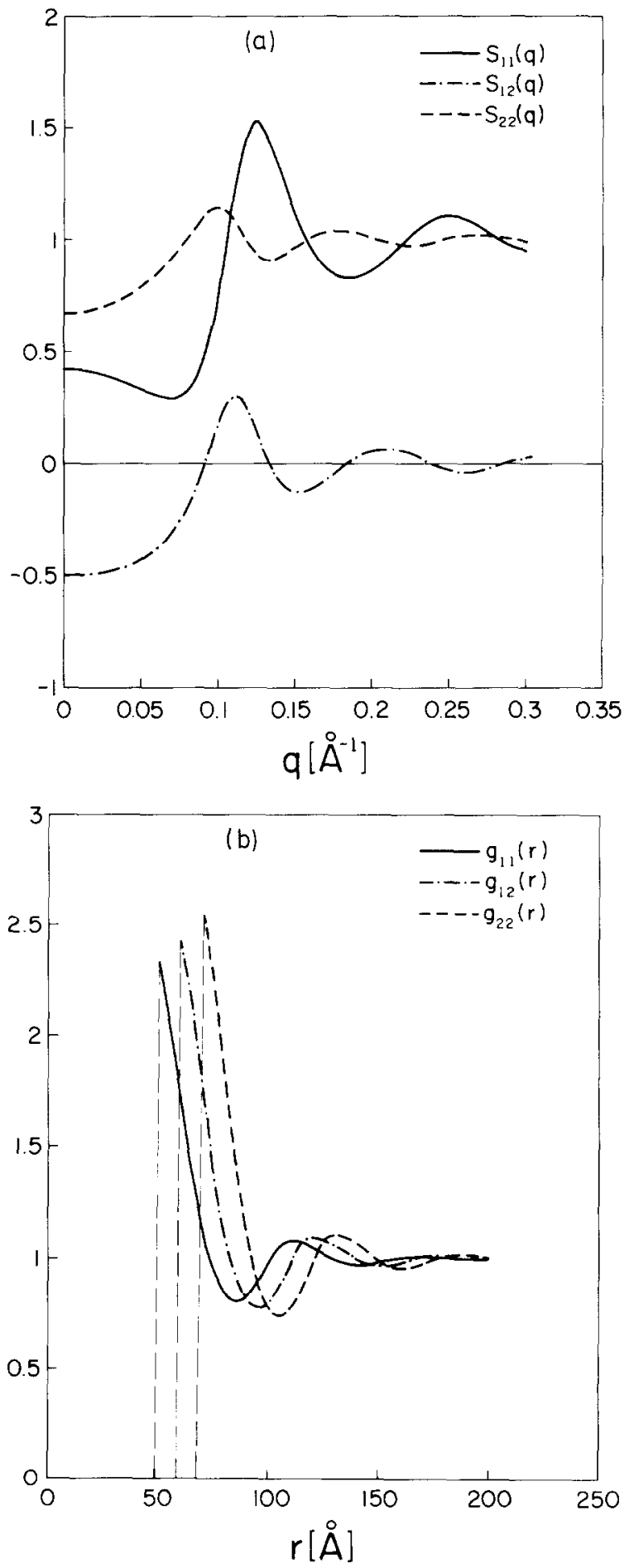
us mention, however, that under some special conditions, the MSA for Yukawa mixtures could lead to other un-physical artifacts, not observed in the monodisperse case. For example, if in a binary mixture one of the species is uncharged (i.e., $A_{1}=0$ ), and the other is highly or even moderately charged, the possibility exists that the contact value of the radial distribution functions are positive, but with the first minimum becoming negative. A simple example is provided by the limiting case in which the first species is uncharged and the second, charged species, is present at infinite dilution. In this case, one can show that

$$
g_{22}^{\mathrm{MSA}}(r)=g_{22}^{\mathrm{PY}}(r)-\left(A_{2}\right)^{2} \frac{\mathrm{e}^{-z r}}{r}, \quad r>\sigma_{2},
$$

for the MSA radial distribution function of the tracer spheres. $g_{22}^{\mathrm{PY}}(r)$ is the Percus-Yevick radial distribution function of the corresponding pure hardsphere system (where also $A_{2}=0$ ). The function $g_{22}^{\mathrm{PY}}(r)$ is positive, with a contact value larger than one. For a sufficiently small coupling amplitude $A_{2}$, $g_{22}^{\mathrm{MSA}}(r)$ is positive at and in a neighborhood of the contact distance $\sigma_{2}$. If now the screening parameter $z$ happens to be small, $g_{22}^{\text {MSA }}(r)$ is observed to be negative in a neighborhood around its first minimum, albeit it is positive around the contact distance. These artifacts, however, are not expected to occur in parameter ranges typical of charged micellar solutions or suspensions of highly charged macroions, which are the systems we have in mind in terms of the practical applications of our results.

As a final remark, let us mention that the practical use of the MSA structure factors of model Yukawa mixtures, in the interpretation of experimental measurements, does require the definition of a precise connection between the model parameters $\mathrm{A}_{\alpha}$ and $z$ and the actual experimental values of the macroparticle charges and ionic strength. Such a connection can be provided by the DLVO potential, as in eq. (1.2), in which case one first determines the Yukawa parameters $A_{\alpha}$ in terms of the given experimental charges $Z_{\alpha}^{\exp }$ using this equation, and setting $z=\kappa^{\text {exp }}$, the corresponding experimental screening constant. Then, the structure factors are evaluated in the manner indicated here, thus resulting in some accessory PM charges, $Z_{\alpha}$, with no particular physical meaning.

Fig. 1. (a) Partial static structure factors $S_{\alpha \beta}(q)$, and (b) radial distribution functions $g_{\alpha \beta}(r)$ calculated within the mean spherical approximation for a hard-sphere plus Yukawa binary mixture with diameters $\sigma_{1}=50 \AA, \sigma_{2}=70 \AA$, number concentrations $n_{1}=4.03 \times 10^{18} \mathrm{~cm}^{-3}, n_{2}=5.35 \times$ $10^{17} \mathrm{~cm}^{-3}$. The interaction potentials $u_{\alpha \beta}$ are given by eq. (1.1), with $A_{1}=54.14 \AA^{1 / 2}, A_{2}=$ $166.96 \AA^{1 / 2}$ and $z=6.77 \times 10^{-2} \AA^{-1}$. These model parameters correspond to a concentrated micellar solution; no rescaling is needed. 
Alternatively, Belloni [12] has suggested, in the context of monodisperse suspensions in the absence of added salt, that this connection be defined by the effective pair potential resulting from the contraction procedure, i.e., by eqs. (2.15)-(2.17). In this case, the primitive model charges $Z_{\alpha}$ are assumed to be the experimental charges, and the MSA macroion-macroion radial distribution functions of the effective Yukawa system with coupling parameters given by eqs. (3.4)-(3.5) are hence given directly by the primitive model MSA solution. Although this suggestion seems to render the Yukawa mixture model completely unnecesary, Belloni also notes that this, or the DLVO definition of an effective Yukawa potential, is still the most useful concept when the MSA correlation functions become negative at contact, since no fully consistent prescription has been proposed to "rescale" the primitive model. Thus, for dilute suspensions of highly charged macroparticles, the effective Yukawa pair potential (within the DLVO, or within Belloni's definition), along with the rescaled mean spherical approximation, continues to be the simplest approach to the structural properties of strongly repulsive systems. The following sections defines the manner to implement the rescaled mean spherical approximation in the casc of mixtures.

\section{Rescaled mean spherical approximation for Yukawa mixtures}

The contact values of the radial distribution function of the binary Yukawa system in fig. (1b) happened to be all positive. It is well known that in strongly coupled systems the MSA gives rise to unphysical negative values of some, or all, of the radial distribution functions in a neighborhood around the contact distance. To overcome these features, Hansen and Hayter [1] provided physical arguments for one-component Yukawa systems leading to the well-known rescaled mean spherical approximation (RMSA). They implemented their rescaling procedure using the analytic MSA solution given by Hayter and Penfold [21], whereas Nägele et al. [2] used, instead, the equivalent MSA solution of Hoye and Blum [22], which is simpler and more compact than that of Hayter and Penfold. Here we extend Hansen and Hayter's MSA rescaling arguments to hard-sphere plus Yukawa mixtures. Let us mention, however, that a particular case of this extension (a binary mixture in the limit of vanishing concentration of one of the species) has been previously discussed by Nägele et al. [23], this being the only precedent of the full extension described here.

The rescaling argument is the following $[1,2,23]$ : consider a dilute $m$ - 
component hard-sphere plus Yukawa mixture of spheres with purely repulsive interactions (that is, all coupling amplitudes $A_{\alpha}$ are of the same sign). Let us assume in addition that the Yukawa tails $A_{\alpha} A_{\beta} \mathrm{c}^{-z r} / r$ happen to have values at hard-sphere contact, $A_{\alpha} A_{\beta} \mathrm{e}^{-z \sigma_{\alpha \beta} / \sigma_{\alpha \beta}}$, which are very much larger than 1 , so that two particles (of species $\alpha$ and $\beta$ ) will virtually never get closer than a certain distance $\sigma_{\alpha \beta}^{\prime}>\sigma_{\alpha \beta}$. As a result, the radial distribution functions $g_{\alpha \beta}(r)$, besides being strictly zero for $r \leqslant \sigma_{\alpha \beta}$, will remain vanishingly small within an interval $\sigma_{\alpha \beta}<r \leqslant \sigma_{\sigma \beta}^{\prime}$, i.c., they do not exhibit any hard-spherc discontinuity at $r=\sigma_{\alpha \beta}$. The effective distances of closest approach $\sigma_{\alpha \beta}^{\prime}$, and in fact the full radial distribution functions $g_{\alpha \beta}\left(r ; \sigma_{1}, \ldots, \sigma_{m}, A_{1}, \ldots, A_{m}, z\right)$, are then only dependent on the Yukawa parameters $A_{\alpha}$ and $z$ and on the thermodynamic state (i.e., on $n_{1}, \ldots n_{m}$ and $T$ ), and do not depend on the hard-sphere diameters $\sigma_{\alpha}$. As a consequence, the same radial distribution functions will describe the structure of a family of systems with the same Yukawa tails and at the same state, but with different hard-sphere diameters, provided that such diameters are such that $\sigma_{\alpha \beta} \leqslant \sigma_{\alpha \beta}^{\prime}$. Thus, we may choose from this family the system with the largest hard-sphere diameters, which we shall denote by $\sigma_{\alpha}^{\prime}$, to represent the structural properties of all the systems in the family, so that we can write

$$
\begin{aligned}
& g_{\alpha \beta}\left(r ; \sigma_{1}, \ldots, \sigma_{m}, A_{1}, \ldots, A_{m}, z\right) \\
& \quad \approx g_{\alpha \beta}\left(r ; \sigma_{1}^{\prime}, \ldots, \sigma_{m}^{\prime}, A_{1}, \ldots, A_{m}, z\right) .
\end{aligned}
$$

Of course, $\sigma_{\alpha}^{\prime}$ must be such that $\frac{1}{2}\left(\sigma_{\alpha}^{\prime}+\sigma_{\beta}^{\prime}\right)$ is identical to the distance of closest approach $\sigma_{\alpha \beta}^{\prime}$ (which only depends on $A_{1}, \ldots, A_{m}$, and $z$ ). The conditions determining $\sigma_{\alpha \beta}^{\prime}$ may then be written as

$$
g_{\alpha \alpha}\left(r=\sigma_{\alpha}^{\prime}+; \sigma_{1}^{\prime}, \ldots, \sigma_{m}^{\prime}, A_{1}, \ldots, A_{m}, z\right)=0 \quad(\alpha=1,2, \ldots, m) .
$$

Thus, if we knew the functions $g_{\alpha \beta}\left(r=\sigma_{\alpha}+; \sigma_{1}, \ldots, \sigma_{m}, A_{1}, \ldots, A_{m}, z\right)$, eq. (4.2) would constitute a set of $m$ equations for the $m$ unknowns $\sigma_{\alpha}^{\prime}$. It is at this point where the mean spherical approximation enters. As it happens, the MSA provides indeed a very reasonable approximation for the structural properties of the representative system, i.e., that with hard-sphere diameters $\sigma_{\alpha}^{\prime}$, although it may be a rather deficient approximation for the original system. Thus, we approximate eq. (4.2) by

$$
g_{\alpha \alpha}^{\mathrm{MSA}}\left(r=\sigma_{\alpha}^{\prime}+; \sigma_{1}^{\prime}, \ldots, \sigma_{m}^{\prime}, A_{1}, \ldots, A_{m}, z\right)=0 \quad(\alpha=1,2, \ldots, m) .
$$


Furthermore, since the accuracy of the MSA radial distribution functions for the effective system is not restricted only to the contact region, we use this fact to approximate the rhs of eq. (4.1). The resulting approximation for the radial distribution functions of the original system is what we define as the rescaled mean spherical approximation (RMSA). Thus, in summary, the rescaled mean spherical approximation is defined as

$$
\begin{aligned}
& g_{\alpha \beta}^{\mathrm{RMSA}}\left(r ; \sigma_{1}, \ldots, \sigma_{m}, A_{1}, \ldots, A_{m}, z\right) \\
& \quad \equiv g_{\alpha \beta}^{\mathrm{MSA}}\left(r ; \sigma_{1}^{\prime}, \ldots, \sigma_{m}^{\prime}, A_{1}, \ldots, A_{m}, z\right)
\end{aligned}
$$

where the effective diameters are first determined solving eq. (4.3).

This rescaling prescription can now be combined with the analytic solution of the MSA for the $m$-component Yukawa system based on the correspondence with the MSA solution of a $(m+1)$-component primitive model, explained in the previous section. The resulting algorithm to calculate the structure of the $m$-component Yukawa mixture with given hard-sphere diameters $\sigma_{\alpha}$ and Yukawa parameters $A_{r}$ and $\mathrm{z}$, may be summarized as follows: for these Yukawa parameters, solve eqs. (3.4), (3.5) for the primitive model charge numbers $Z_{\alpha}$ and $Z_{s}$, keeping the same macroion concentrations and hardsphere diameters as given for the Yukawa mixture. Next, check if all the corresponding macroion-macroion radial distribution functions at contact, $g_{\alpha<r}^{\mathrm{MSA}}\left(\sigma_{\alpha r}+\right)$, are negative. If yes, solve the following $(2 m+1)$ equations (the $n_{\alpha}$ being kept fixed):

$$
\begin{aligned}
& g_{\alpha \alpha}^{\mathrm{MSA}(\mathrm{PM})}\left(r=\sigma_{\alpha}^{\prime}+; \sigma_{1}^{\prime}, \ldots, \sigma_{m}^{\prime}, Z_{1}^{\prime}, \ldots, Z_{m}^{\prime}, Z_{s}^{\prime}\right)-0, \\
& K_{\alpha}\left(\sigma_{1}^{\prime}, \ldots, \sigma_{m}^{\prime}, Z_{1}^{\prime}, \ldots, Z_{m}^{\prime}, Z_{s}^{\prime}\right)=A_{\alpha}
\end{aligned}
$$

and

$$
\kappa\left(Z_{1}^{\prime}, \ldots, Z_{m}^{\prime}, Z_{s}^{\prime}\right)=z
$$

for the $(2 m+1)$ unknown parameters $\sigma_{\alpha}^{\prime}, Z_{\alpha}^{\prime}$ and $Z_{\mathrm{s}}^{\prime}$ of the corresponding $(m+1)$-component PM. The functions $g_{\alpha \alpha}^{\mathrm{MSA}(\mathrm{PM})}$ are the MSA contact values of the PM (see eq. (12) of ref. [10]), and the functions $K_{c \gamma}$ and $\kappa$ are, once again, those defined in eqs. (2.16) and (2.7) respectively. Notice that here also the counterion parameters $n_{\mathrm{s}}$ and $Z_{\mathrm{s}}^{\prime}$ are trivially expressible in terms of $Z_{\mathrm{l}}^{\prime}, \ldots, Z_{m}^{\prime}$ via eq. (4.7) and the electroneutrality condition $n_{\mathrm{s}} Z_{\mathrm{s}}^{\prime}=$ $-\sum_{y=1}^{m} n_{\gamma} Z_{\gamma}^{\prime}$, which means that only the $2 m$ equations $(4.5),(4.6)$ have to be solved numerically for the $2 m$ unknowns $\sigma_{\alpha}^{\prime}\left(>\sigma_{\alpha}\right)$ and $Z_{\alpha}^{\prime}$. Then the radial 
distribution functions $g_{\alpha \beta}^{\mathrm{MSA}}(r)$ required in eq. (4.4) are calculated using Blum's PM solution [8-11], i.e., eq. (4.4) can also be written as

$$
\begin{aligned}
& g_{\alpha \beta}^{\mathrm{RMSA}}\left(r ; \sigma_{1}, \ldots, \sigma_{m}, A_{1}, \ldots, A_{m}, z\right) \\
& \quad \equiv g_{\alpha \beta}^{\mathrm{MSA}(\mathrm{PM})}\left(r ; \sigma_{1}^{\prime}, \ldots, \sigma_{m}^{\prime}, Z_{1}^{\prime}, \ldots, Z_{m}^{\prime}, Z_{\mathrm{s}}^{\prime}\right) .
\end{aligned}
$$

It is not the aim of this paper to report an extensive comparison of the RMSA with other integral equations (HNCA, etc.), or against computer simulation data. Such a comparison has been made in the case of monodisperse systems [1-3], and it will be done for mixtures in a forthcoming paper. However, for illustrative purposes, let us discuss the application of our RMSA approach to calculate the structural properties of a dilute binary mixturc of highly charged polystyrene spheres. We choose the system parameters in our illustration in such a way to compare with available computer simulation results. Thus, we take the Yukawa parameters to have the values $A_{1}=$ $672.88 \AA^{1 / 2}, A_{2}=961.26 \AA^{1 / 2}, z=6.8 \times 10^{-4} \AA^{-1}$, and the number concentrations $n_{1}=2.656 \times 10^{12} \mathrm{~cm}^{-3}$ and $n_{2}=5.344 \times 10^{12} \mathrm{~cm}^{-3}$. In addition, we set both hard-sphere diameters equal to $500 \AA$, i.e., $\sigma_{1}=\sigma_{2}=500 \AA$. These parameters correspond to a dilute mixture of highly charged polystyrene spheres in water at room temperature. The Yukawa amplitudes above would correspond, according to eq. (3.6), to DLVO charges of $Z_{1}^{\text {DLVO }}=247.7$ and $Z_{2}^{\mathrm{DLVO}}=353.9$. Alternatively, if we were to use Belloni's connection between Yukawa parameters and macroion charges, i.e., if we solve eqs. (3.4), (3.5) for $Z_{1}$ and $Z_{2}$ with the Yukawa parameters $A_{1}, A_{2}$ and $z$ above, we would determine $Z_{1}=248.3$ and $Z_{2}=355.0$. Clearly, in this illustrative case the quantitative difference between these two sets of charge numbers is rather small.

The straightforward use of the MSA for this Yukawa mixture leads to negative contact values of all the radial distribution functions. In fact we find $g_{11}^{\mathrm{MSA}(\mathrm{PM})}\left(\sigma_{1}+\right)=-206.6, g_{12}^{\mathrm{MSA}(\mathrm{PM})}\left(\sigma_{12}+\right)=-295.7$, and $g_{22}^{\mathrm{MSA}(\mathrm{PM})}\left(\sigma_{2}+\right)=$ -423.1 . Thus, rescaling is necessary. The application of our algorithm requires the solution of eqs. (4.5)-(4.7) for the cffective primitive-model parameters $\sigma_{1}^{\prime}, \sigma_{2}^{\prime}, Z_{1}^{\prime}, Z_{2}^{\prime}$ and $Z_{\mathrm{s}}^{\prime}$, which are found in this manner to have the values $\sigma_{1}^{\prime}=3671.8 \AA, \quad \sigma_{2}^{\prime}=4247.3 \AA, \quad Z_{1}^{\prime}=137.95, \quad Z_{2}^{\prime}=172.57$ and $Z_{\mathrm{s}}^{\prime}=-3.97$. Notice that the increase in the diameters has been compensated with a corresponding decrease in the charges in order to keep the Yukawa amplitudes to be the same as in the original system. This is also and more easily appreciated from the DLVO connection in eq. (3.6) between coupling amplitudes, charges, and diameters. Finally, the RMSA macroion-macroion radial distribution functions for our original Yukawa mixture are given, 
according to eq. (4.8), by the macroion-macroion radial distribution functions of this primitive model (with, of course, the same macroion number concentrations $n_{1}$ and $n_{2}$ as above). The resulting radial distribution functions are presented in fig. 2, which also include for comparison the computer simulation data of Kremer et al. [24, 25]. Clearly, although there are evident quantitative discrepancies in the height of the first maximum of $g_{\alpha \beta}(r)$, the overall comparison seems to be quite satisfactory given the simplicity of our approach. Furthermore, the extents of these discrepancies are not much more serious than they are known to be in the monodisperse case under conditions of strong electrostatic couplings [2]. Let us emphasize, however, that the RMSA determination of the effective distance of closest approach, $\sigma_{\alpha \beta}^{\prime}$, as well as the prediction of the location of the maxima and minima of $g_{\alpha \beta}(r)$, are found to be in full quantitative agreement with the computer simulation results, as it can be observed in fig. 2.

Let us also comment that the rescaling procedure is based on the condition that $g_{\alpha \alpha}\left(\sigma_{\alpha}^{\prime}+\right)=0$, but no similar condition is imposed a priori on the cross-correlation functions $g_{\alpha \beta}(r)$ (with $\alpha \neq \beta$ ) at $r=\frac{1}{2}\left(\sigma_{\alpha}^{\prime}+\sigma_{\beta}^{\prime}\right)$. Thus, we should not expect $g_{\alpha \beta}\left(\sigma_{\alpha \beta}^{\prime}+\right)$ to be exactly zero. However, a noticeable departure of these quantities from zero could probably indicate overall inaccuracies of the RMSA results. Hence, monitoring the value of this quantity

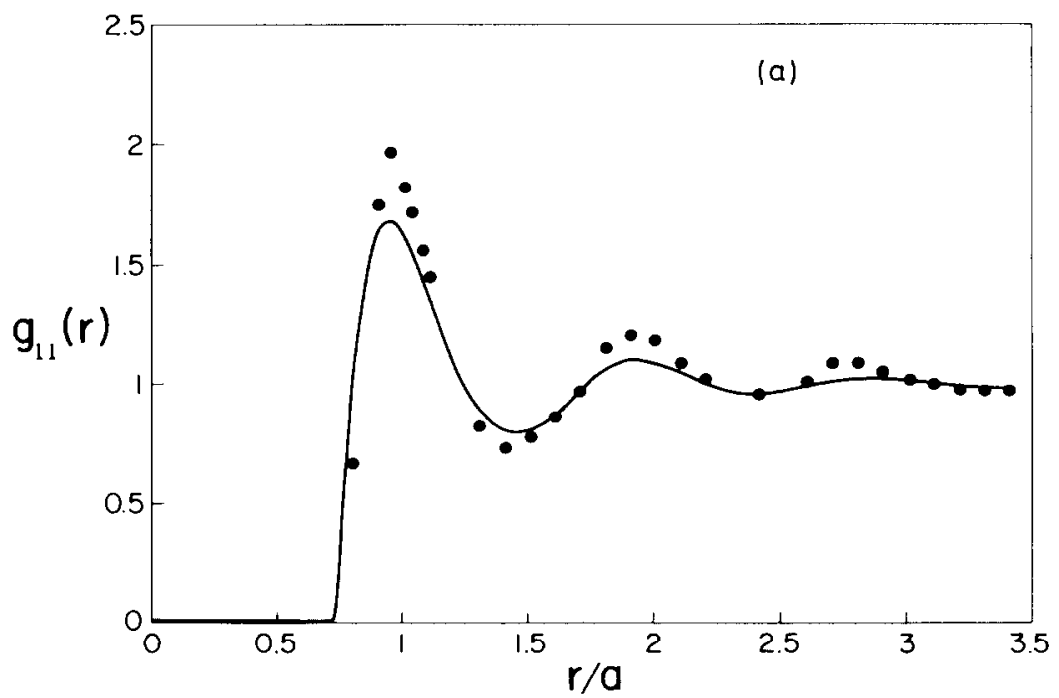

Fig. 2. (a)-(c) Radial distribution functions $g_{\alpha \beta}(r)$ calculated within the rescaled mean spherical approximation for a hard-sphere plus Yukawa binary mixture with parameters $\sigma_{1}=\sigma_{2}=500 \AA$, $n_{1}=2.66 \times 10^{12} \mathrm{~cm}^{-3}, \quad n_{2}=5.34 \times 10^{12} \mathrm{~cm}{ }^{3}, \quad z=6.8 \times 10^{-4} \AA^{-1}, \quad A_{1}=672.9 \AA^{1 / 2}$, and $A_{2}=$ $961.3 \AA^{1 / 2}$. The radial distance is scaled with the mean interparticle distance $a=\left(n_{1}+n_{2}\right)^{-1 / 3}$. The circles are the computer simulation results of Kremer et al. [24]. 

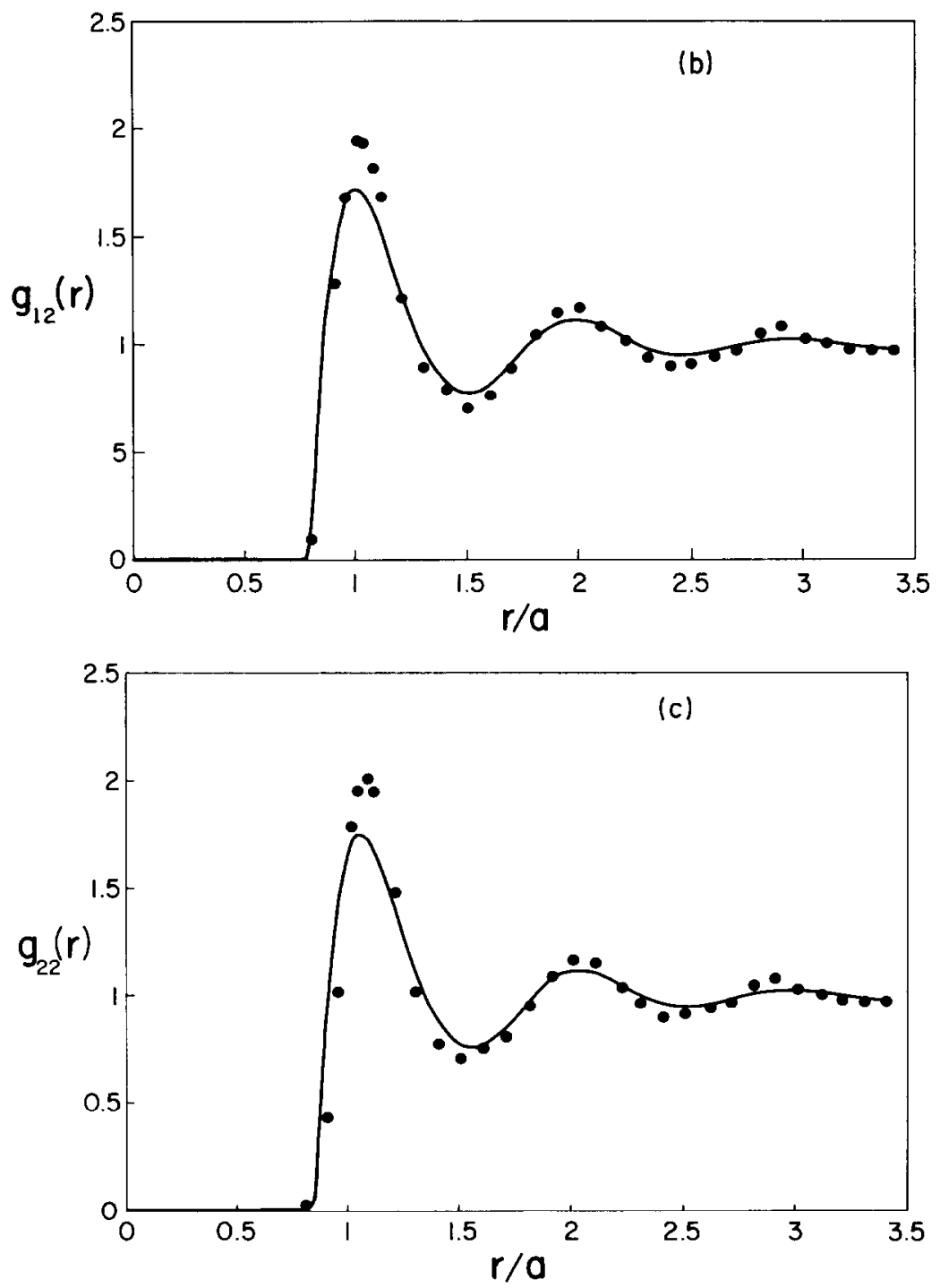

Fig. 2 (cont.).

might be an advisable suggestion during the use of our method. In the case of the illustrative example above, we find $g_{12}\left(\sigma_{12}^{\prime}+\right)=0.002$, which is, of course, a completely acceptable value. For most systems of this type, we found similar results, although we also observed that stronger asymmetries in the coupling parameters $A_{\alpha}$ could lead to appreciable departures of $g_{12}\left(\sigma_{12}^{\prime}+\right)$ from zero. To quantify this observation for systems related to that in our illustration, fig. 3 shows $g_{12}\left(\sigma_{12}^{\prime}+\right)$ as a function of the coupling asymmetry parameter, $A_{2} / A_{1}$. 


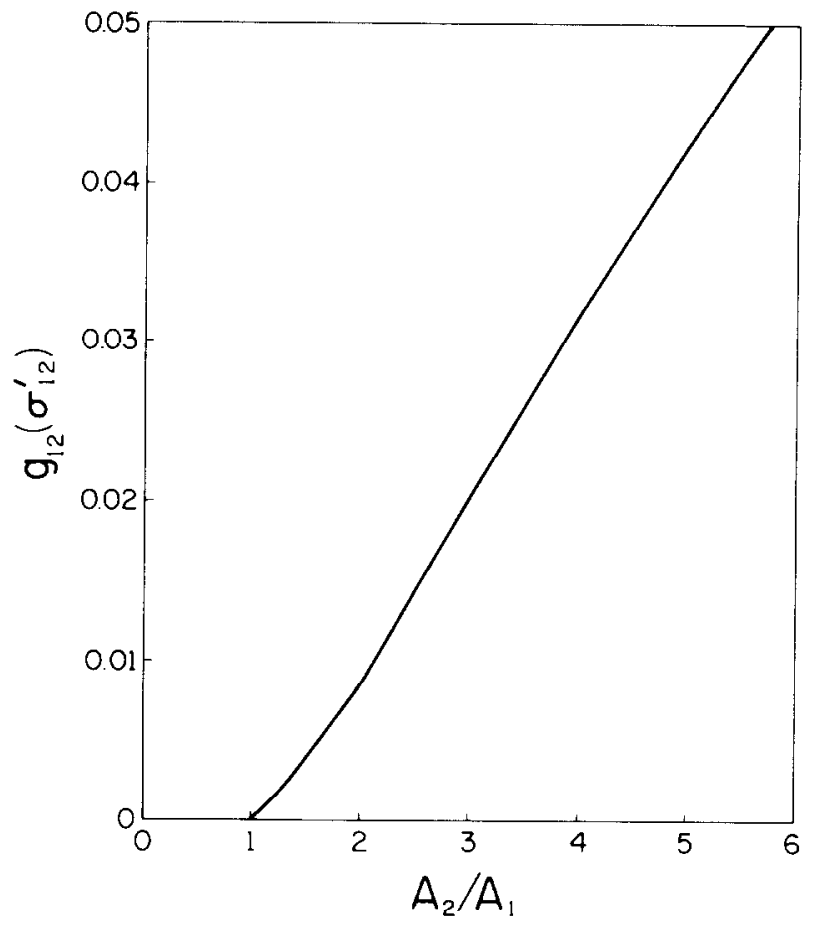

Fig. 3. Cross radial distribution function $g_{12}(r)$ at $r=1_{2}^{1}\left(\sigma_{1}^{\prime}+\sigma_{2}^{\prime}\right)$ obtained from the rescaled mean spherical approximation for the same parameters as in fig. 2. except that $A_{2}$ is being varied from $A_{2}=A_{1}$ to $A_{2}=6 A_{1}$.

These results correspond to the same system as in fig. 2, except that the coupling amplitude $A_{2}$ was varied from $A_{2}=A_{1}$ to $A_{2}-6 A_{1}$ (the system in fig. 2 corresponds to $A_{2} / A_{1} \simeq 1.4$ ). These results illustrate the fact that even under rather severe conditions of charge asymmetry, the discontinuity of $g_{12}^{\text {RMSA }}(r)$ at $r=\sigma_{12}^{\prime}$ is still rather small compared with the relatively large values that $g_{12}(r)$ must attain at slightly larger distances, namely, at its first maximum. Plotted in a similar manner we also show in fig. 4 the corresponding effective diameters $\sigma_{\alpha}^{\prime}$ and the corresponding primitive model charge numbers $Z_{\alpha}^{\prime}$. Notice that as the asymmetry increases, a larger effective diameter corresponds to the more highly charged species. We can also see that the increase in $A_{2}$ also needs a corresponding increase in the effective charge number $Z_{2}^{\prime}$. On the other hand, for the species whose coupling amplitude, $A_{1}$, remained unchanged, the increase in $A_{2}$ leads to a decrease in its effective diameter $\sigma_{1}^{\prime}$, but also to a rather mild change in its charge number $Z_{1}^{\prime}$. Let us mention that for the conditions in figs. 3 and 4 , we found that $\phi_{\mathrm{T}}^{\prime} \equiv$ 


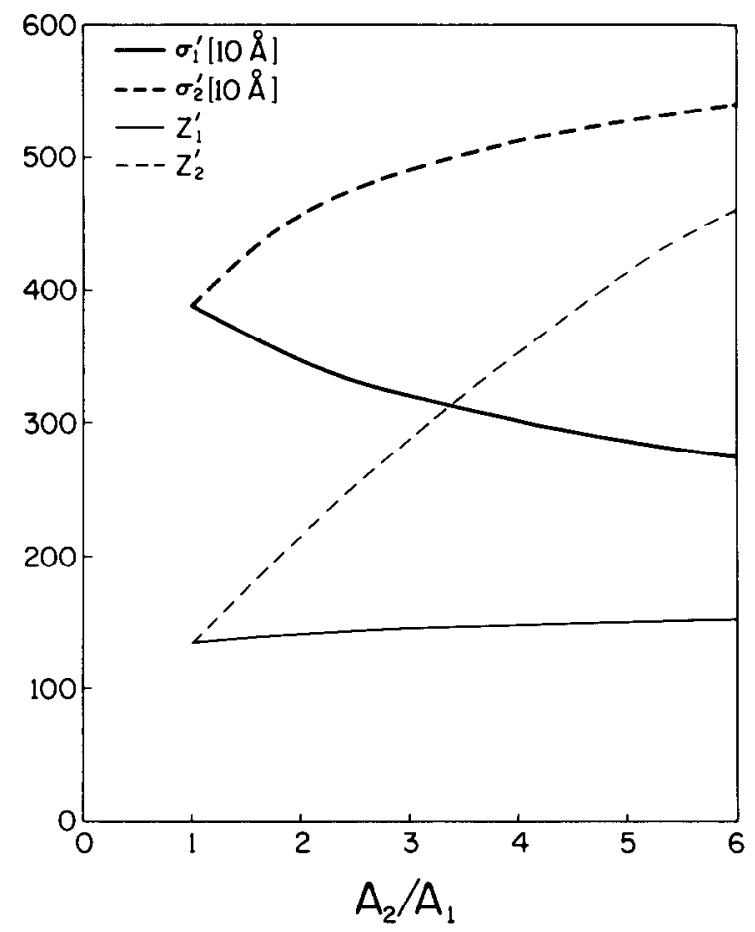

Fig. 4. Hard-sphere diameters $\sigma_{1}^{\prime}$ and $\sigma_{2}^{\prime}$, and charge numbers $Z_{1}^{\prime}$ and $Z_{2}^{\prime}$ of the primitive model corresponding to the rescaled binary Yukawa mixture with the same parameters as in fig. 3 .

$\frac{1}{6} \pi \Sigma_{y=1}^{2} n_{\gamma}\left(\sigma_{\gamma}^{\prime}\right)^{3}$, the effective volume fraction, was always below 0.6. In particular, for the system in fig. 2 we found that $\phi_{\mathrm{T}}^{\prime}=0.283$. As a reference, a monodisperse hard-sphere system freezes at a volume fraction of approximately 0.49 .

As a final remark, let us notice the following differences of our RMSA procedure for Yukawa mixtures with respect to the one-component rescaling procedure of Hansen and Hayter: the rescaling procedure is, in the monodisperse case, almost always applicable, for parameter sets within the physically reasonable regimc. Our cxtended rescaling procedure, however, may be more restricted in its applicability. First, it works only in mixtures with purely repulsive interaction, i.e., $A_{\alpha} A_{\beta}>0$ for all $\alpha, \beta \in\{1, \ldots, m\}$. Second, if one or more of the MSA contact values $g_{\alpha \alpha}\left(\sigma_{\alpha}+\right)$ at the physical contact distance $\sigma_{\alpha}$ happen to be positive, then our procedure is not applicable, and third, large asymmetries in the coupling amplitudes may imply unphysical discontinuities in the RMSA cross radial distribution functions, i.e. $g_{\alpha \beta}^{\text {RMSA }}\left(\sigma_{\alpha \beta}^{\prime}+\right)$ may be appreciably different from zero for $\alpha \neq \beta$. However, the general ideas behind the rescaling arguments do have in mind systems with strong electrostatic 
repulsions, of the type that has been studied rather extensively in the last years in the monodisperse case. Mixtures of such model systems will most likely fall in the range of applicability of our approach.

\section{Conclusions}

In this work we have introduced a scheme to solve the mean spherical approximation for Yukawa mixtures with factorized coupling parameters. This scheme is based on the transformation of the original problem into the equivalent, but much easier, problem of determining the MSA structure functions of a corresponding primitive model system with point-like counterions. The resulting algorithm was illustrated by calculating the structure functions of a binary Yukawa system with parameters typical of a concentrated binary mixture of charged micelles.

In a second step, this algorithm for solving the MSA in Yukawa mixtures was combined with the rescaling procedure for those cases in which the contact value of the MSA macroion-macroion radial distribution functions were all negative. The resulting rescaled mean spherical approximation allows for the calculation of the structure of mixtures of highly charged macroions, like polystyrene spheres in water. For such systems, the MSA itself predicts unphysical radial distribution functions. As an illustration, the RMSA structure functions of a bidisperse Yukawa mixture with strong interparticle repulsion were calculated here. They were found to compare well with computer simulation data. Comments on the range of applicability of the RMSA for mixtures were given: It applies only to purely repulsive Yukawa pair potentials, i.e. $A_{\alpha} A_{\beta}>0$, and the $g_{\alpha \beta}^{\text {RMSA }}(r)$ for $\alpha \neq \beta$ are expected to exhibit no appreciable discontinuity only for mixtures with not too broad asymmetries in the coupling amplitudes. Furthermore, the rescaling procedure does not apply if at least one of the partial radial distribution functions shows, before rescaling, a positive discontinuity at the physical contact distance.

In spite of these limitations, the RMSA for Yukawa mixtures is expected to be quite useful for the calculation of static structure functions. The merit of our RMSA scheme is its analytic simplicity, which makes it easy to apply for mixtures with more than two species. This makes it feasible to use the RMSA scheme for the investigation of polidispersity effects in macroion mixtures. In addition, the RMSA scheme allows for a fast calculation of partial structure factors over an extended range of wavenumbers. This is important for the calculation of dynamic properties in mixtures. We are at present using our RMSA structure factors in the calculation of self-diffusion properties in binary suspensions of highly charged polystyrene spheres suspended in water, whose 
static and dynamic properties are currently under experimental investigation [26-28].

\section{Acknowledgements}

The authors acknowledge the support of the Consejo Nacional de Ciencia $y$ Tecnología (CONACyT, Mexico). G. Nägele expresses his gratitude to the Deutsche Forschungsgemeinschaft (DFG) for support and to Professor A.Z. Akcasu for the kind hospitality and useful discussions during his stay at the University of Michigan at Ann Arbor. H. Ruiz-Estrada acknowledges the Consejo del Sistema Nacional de Educación Tecnológica (COSNET, Mexico) for a graduate fellowship. The authors also acknowledge R. Krause, Univ. of Konstanz, F.R.G., for carefully reading our manuscript.

\section{References}

[1] J.P. Hansen, J.B. Hayter, Mol. Phys. 46 (1982) 651.

[2] G. Nägele, M. Medina-Noyola and R. Klein, Physica A 149 (1988) 123.

[3] B. Svenson, B. Jönsson, Mol. Phys. 50 (1983) 489.

[4] L. Blum and J.S. Hoye, J. Stat. Phys. 19 (1978) 317.

L. Blum, J. Stat. Phys. 22 (1980) 661.

[5] E. Arrieta, C. Jedrzejec and K.N. March, J. Chem. Phys. 86 (1987) 3607.

[6] G. Pastore, Mol. Phys. 63 (1988) 731.

[7] M. Ginoza, J. Phys. Soc. Jpn. 55 (1986) 95.

[8] L. Blum, Mol. Phys. 30 (1975) 1519.

[9] L. Blum and J.S. Hoye, J. Phys. Chem. 81 (1977) 1311.

[10] K. Hiroike, Mol. Phys. 33 (1977) 1519.

[11] K. Hiroike, J. Phys. Soc. Jpn. 27 (1969) 1415.

[12] L. Belloni, J. Chem. Phys. 85 (1986) 519.

[13] M. Medina-Noyola, J. Chem. Phys. 77 (1982) 1428.

[14] G. Nägele, R. Klein and M. Medina-Noyola, J. Chem. Phys. 83 (1985) 2560.

[15] G. Senatore and L. Blum, J. Phys. Chem. 89 (1985) 2676.

[16] B. Beresford-Smith, D.Y.C. Chan and D.J. Mitchell, J. Colloid Interf. Sci. 105 (1985) 216.

[17] S. Khan and D. Ronis, Mol. Phys. 60 (1987) 637.

[18] D. Bratko, E.Y. Sheu, and S.-H. Chen, Phys. Rev. A 35 (1987) 4359.

[19] M. Medina-Noyola and D.A. McQuarrie, J. Chem. Phys. 73 (1980) 6279.

[20] S.A. Adelman, J. Chem. Phys. 64 (1976) 724.

[21] J.B. Hayter and J. Penfold, J. Chem. Soc. Faraday Trans. 1, 77 (1981) 1851.

[22] J.S. Hoye and L. Blum, J. Stat. Phys. 16 (1977) 399.

[23] G. Nägele, M. Medina-Noyola, J.L. Arauz-Lara and R. Klein, Progr. Colloid Polym. Sci. 73 (1987) 5 .

[24] K. Kremer, Johannes-Gutenberg-Universität, Mainz, private communication.

[25] K. Kremer, G.S. Grest and M.O. Robbins, J. Phys. A: Math. Gen. 20 (1987) L181.

[26] X. Qiu, D. Ou-Yang and P.M. Chaikin, J. Phys. (Paris) 49 (1988) 1043.

[27] H. Härtl and H. Versmold, J. Chem. Phys. 80 (1984) 1387.

[28] R. Krause and R. Weber, Univ. Konstanz, F.R.G., private communication. 